\title{
Implantation et persistance des souches mucoïdes de Pseudomonas aeruginosa dans les poumons des malades atteints de mucoviscidose
}

La colonisation des poumons par des souches mucoïdes de Pseudomonas aeruginosa est à l'origine des graves complications respiratoires apparaissant à un stade évolué de la mucoviscidose. La forte osmolarité du mucus bronchique des malades semble jouer un rôle dans l'activation de gènes bactériens qui vont diriger la synthèse d'une gangue protectrice d'exopolysaccharides de type alginate ; celle-ci facilitera l'adsorption du pathogène, le protègera des antibiotiques, des anticorps et des macrophages et empêchera son élimination par le système mucociliaire. Ainsi $P$. aeruginosa continuera-t-il à sécréter différentes substances toxiques accroissant les lésions tissulaires et des polysaccharides qui augmenteront encore la viscosité des sécrétions bronchiques, et donc l'insuffisance respiratoire des malades.

\section{Michèle}

\section{Pennacino-Sauvage} Christian Hulen

\section{ADRESSE}

M. Pennacino-Sauvage : stagiaire de recherche, boursière de l'Association française de lutte contre la mucoviscidose. C. Hulen : maître de conférences, à l'université Paris XI. Cercoa-Cnrs, 2 à 8, rue Henri-Dunant, 94320 Thiais,

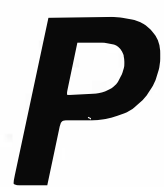
seudomonas aenuginosa, bactérie saprophyte opportuniste, est actuellement à l'origine d'un grand nombre d'infections chez l'homme, les insectes et les plantes. Ce microorganisme présente une résistance naturelle à un certain nombre d'antibiotiques et une capacité incomparable à s'adapter et à survivre dans des environnements hostiles. Depuis plus de vingt ans, le milieu hospitalier du monde occidental s'est vu coloniser par Pseudomonas aeruginosa, responsable d'un nombre croissant de pneumonies ou de bactérié- mies chez les malades sous traitement immunosuppresseur, ou chez les grands brûlés.

La pathogénicité de Pseudomonas aeruginosa est liée à la capacité du microorganisme de s'adsorber sur un grand nombre de supports (verre, métal, polymères...) et de tissus épithéliaux, et de sécréter des quantités importantes de produits ayant une activité nécrosante sur ces tissus (phospholipase, protéases et exotoxines).

Il existe, à ce jour, des souches particulièrement résistantes à un nombre croissant d'antibiotiques et qui pré- 
sentent un caractère mucoïde par fabrication d'exopolysaccharides de type alginate (polysaccharides constitués d'acides uroniques). Les formes mucoïdes de Pseudomonas aeruginosa sont rencontrées dans certains cas d'infections urinaires ou d'otites, et colonisent surtout les poumons des malades atteints de mucoviscidose. Cette infection particulière, persistante, représente une des phases les plus sévères de la maladie. Chez les enfants atteints de mucoviscidose, l'infection précoce des voies respiratoires par Pseudomonas aeruginosa est actuellement la cause majeure de mortalité.

\section{La mucoviscidose}

La mucoviscidose est la maladie héréditaire la plus fréquente dans les populations nord-européennes issues de la branche caucasienne. Elle est la conséquence d'une mutation sur un seul gène situé sur le bras long du chromosome 7. Cette mutation récessive est présente actuellement à une fréquence d'environ $1 / 16$ dans les populations anglo-saxonnes et $1 / 20$ dans la population française, ce qui donne en moyenne un enfant homozygote pour environ 2000 naissances [1]. La gravité de la maladie varie d'un sujet à un autre, mais elle ne reste jamais sans effet.

Les résultats obtenus récemment par l'emploi des techniques de la génétique inverse ont permis la localisation fine, puis le clonage du gène de la mucoviscidose [2]. Ce gène code pour une protéine membranaire (CFTR, cystic fibrosis transmembrane conductance regulator) impliquée dans le transport ou la régulation du transport des ions chlore à travers la membrane plasmique des cellules des glandes exocrines (pancréas, glandes salivaires et sudoripares) et des cellules des tissus épithéliaux à activité sécrétoire (voies respiratoires) [3].

L'imperméabilité de ces cellules au chlore provoque la production de sécrétions visqueuses, collantes et mal hydratées (l'eau, suivant le mouvement des électrolytes, ne sort pas des cellules), responsables des manifestations symptomatologiques de la mucoviscidose. Cette mauvaise hydratation des sécrétions provoque une obturation des canaux, aboutissant à une dégénérescence du pan- créas, à un accroissement de la taille des glandes et à l'apparition, dans les voies respiratoires inférieures, d'un mucus épais, très visqueux responsable d'une diminution de l'activité ciliaire [4]. Ce défaut dans l'élimination physique du mucus pulmonaire est à l'origine d'une obstruction des petites voies aériennes, cause d'une insuffisance respiratoire et de surinfections bactériennes chroniques, plus ou moins graves, laissant à chaque fois des séquelles définitives dans les tissus.

\section{Les infections pulmonaires}

Les premières infections sont en général provoquées par Staphylococcus aureus et Haemophilus influenzae, pathogènes couramment rencontrés dans les voies respiratoires des enfants. Cependant, leur persistance dans les poumons après une infection aiguë est spécifique des enfants mucoviscidosiques. Au cours de la croissance, grâce à un traitement antibiotique adapté, ces deux espèces bactériennes disparaissent, mais sont souvent remplacées par Pseudomonas aeruginosa qui devient peu à peu l'unique pathogène responsable de la détérioration clinique du malade [5]. Une étude récente, utilisant les techniques d'immuno-histocytologie, a montré que les bactéries étaient adsorbées sur les membranes des bronchioles, mises à nu et attaquées par les toxines bactériennes [6].

La colonisation des malades par Pseudomonas aeruginosa et l'inefficacité des traitements antibiotiques actuels à éliminer totalement ce pathogène sont des obstacles importants à la qualité et à l'espérance de vie des enfants atteints de mucoviscidose.

- Colonisation par Pseudomonas aeruginosa et émergence des souches mucoïdes

La colonisation par Pseudomones aeruginosa des malades atteints de mucoviscidose se fait initialement par adsorption de souches bactériennes non mucoïdes sur la muqueuse buccale, puis par colonisation des voies aériennes supérieures [7]. La salive de ces malades favorise même l'agrégation des bactéries (environ 7 fois plus que celle des personnes saines, [8]). La migration de ces souches vers les voies respiratoires inférieures précède l'émergence du caractère mucoïde et l'établissement des infections chroniques, caractéristiques de l'atteinte pulmonaire.

Il existe un tropisme de Pseudomonas aeruginosa pour l'appareil respiratoire. L'adhérence des bactéries sur les mucines trachéobronchiales se fait par les pilis pour les souches non mucoïdes, puis par les alginates quand les souches deviennent mucoïdes [9].

L'émergence des souches mucoïdes de Pseudomonas aeruginosa est due à des facteurs qui semblent spécifiques du poumon des malades. Le mécanisme du passage de l'état non mucoïde à l'état mucoïde n'est pas encore parfaitement élucidé mais l'on sait qu'il fait intervenir une régulation génétique complexe au niveau du chromosome bactérien, associée à des facteurs externes :

- l'antibiothérapie, nécessaire à l'élimination des autres espèces bactériennes (Staphylococcus aureus et Haemophilus influenzae). Elle est responsable de la sélection, puis de la prédominance de Pseudomonas aeruginosa [10] ;

- l'adhérence préférentielle des souches mucoïdes à l'épithélium trachéal et trachéobronchique, surtout quand les tissus sont endommagés soit par les intubations endotrachéales (permettant l'élimination du mucus), soit par bronchiectasie, soit par le dysfonctionnement mucociliaire [9, 11] ;

- la présence d'une concentration élevée en calcium dans les poumons augmentant les propriétés gélifiantes des alginates et permettant la formation de microcolonies résistantes aux différents systèmes d'élimination [12] ;

- la présence d'une concentration élevée en $\mathrm{NaCl}$, stimulant chez la bactérie la production des enzymes de la biosynthèse des alginates [13].

- Persistance des souches mucoïdes de Pseudomonas aeruginosa dans les voies respiratoires (figure $1, p$. 889) Plusieurs éléments interviennent successivement pour que Pseudomonas aeruginosa soit l'unique pathogène persistant dans les poumons des malades.

- La résistance aux antibiotiques. La gangue muqueuse, constituée 


\section{RÉFÉRENCES}

1. Kitzis A, Warren P, Kaplan JC. Génétique inverse et mucoviscidose. médecine/sciences $1988 ; 4: 151-6$.

2. Rommens JM, Iannuzzi MC, Kerem BS, et al. Identification of the cystic fibrosis gene : chromosome walking and jumping. Science 1989 ; 245 : 1059-65.

3. Riordan JR, Rommens JM, Kerem BS, et al. Identification of the cystic fibrosis gene : cloning and characterization of complementary DNA. Science $1989 ; 245$ : 1066-73.

4. Chartrand SA, Marks MI. Pulmonary infections in cystic fibrosis pathogenesis and therapy. In : Pennington JE, ed. Respiratory Infections: Diagnosis and Management. New York : Raven Press, 1983 : 201-16.

5. Ramphal R, Vishawanath S. Why Pseudomonas the colonisateur and why does it persist? Infection $1987 ; 15: 278-87$.

6. Baltimore RS, Christie CDC, Smith GJW. Immunohistopathologic localization of $P$. aeruginosa in lungs from patients with cystic fibrosis. Am Rev Resp Dis 1989 ; 140 : 1650-61.

7. Woods DE, Iglewski BH, Johanson WG. Host and bacterial factors in the colonization of the respiratory tract. In : Schlessinger D, ed. Microbiology. Washington DC : ASM publications, $1982: 348-52$.

8. Komiyama K, Habbick B, Tumber SK. Whole submandibular and parotid salivamediated aggregation of $P$. aeruginosa in cystic fibrosis. Infect Immun 1989; 57 : 1299-304.

9. Ramphal R, Pier GB. Role of Pseudomonas aenuginosa mucoid polysaccharide in adherence to tracheal cells. Infect Immun 1985; $47: 1-4$.

10. Govan JRW, Fyfe JAM. Mucoids Pseudomonas aeruginosa and cystic fibrosis : resistance of the mucoid form to carbenicillin, flucloxacillin, tobramycin and the isolation of mucoid variants in vitro. I Antimicrobiol Chemoth 1978 ; 4 : 232-240.

11. Pier GB. Pulmonary disease associated with Pseudomonas aeruginosa in cystic fibrosis : current status of the host-bacterium interac-

d'alginates (polymères d'acides uroniques chargés négativement) entourant les bactéries, les protège contre l'action des antibiotiques. La haute force ionique de cette barrière est responsable de l'absence de pénétration des antibiotiques jusqu'à la microcolonie. En particulier, les alginates sont capables de fixer de grandes quantités d'aminoglycosides, généralement actifs sur les souches non mucoïdes de Pseudomonas aeruginosa [14].

- L'inhibition des systèmes d'élimination physique. En règle générale, les bactéries infectant les voies respiratoires sont complexées à des IgA et éliminées dans le mucus bronchique par l'activité ciliaire des cellules épithéliales. L'efficacité de ce système est très diminuée dans les poumons des malades atteints de mucoviscidose. L'association d'un mucus hypervisqueux aux alginates produits par les bactéries mucoïdes inhibe pratiquement toute l'activité ciliaire et entraîne la formation de complexes stagnants [5]. Il faut aussi noter que certaines souches mucoïdes de $P$. aeruginosa sont capables de s'adsorber spécifiquement par leurs alginates sur des cellules de l'épithélium buccal [15] et participent ainsi à la persistance de l'infection.

- L'inhibition du système immunitaire. Les malades atteints de mucoviscidose n'ont pas les signes classiques d'immunosuppression généralement associés aux infections par Pseudomonas aeruginosa. Paradoxalement, la réponse immunitaire est plus importante que chez les autres malades et c'est malgré la présence d'un grand nombre d'anticorps dirigés contre les différentes parties de la bactérie (alginates, LPS, protéines de membrane externe) que la colonisation persiste, ce qui suggère l'existence d'une anomalie dans le fonctionnement de cette réponse immunitaire.

Ramphal et Vishwanath [5] font l'hypothèse que les mucines liées aux protéines bactériennes sont reconnues comme protéines du soi et donc capables d'éviter le système immunitaire. L'absence d'opsonisation de Pseudomonas aeruginosa est peut-être due à cette liaison préférentielle aux mucines et serait une raison de sa sélection comme colonisateur prédominant.
Pseudomonas aeruginosa produit plusieurs facteurs de virulence dont le plus toxique est l'exotoxine $\mathrm{A}$, agissant par ADP ribosylation du facteur d'élongation de la traduction EF2 et inactivant, entre autres, les macrophages.

Parmi les autres toxines produites par la bactérie, l'élastase contribue directement aux lésions tissulaires qui sont aussi favorisées par l'inactivation des composés du complément (C3, C5a) et des IgG [5]. Elle est aussi capable de dégrader les protéines à activité antibactérienne, comme le lysozyme, présentes dans le mucus bronchique [16].

Outre l'élastase bactérienne, une autre élastase d'origine humaine, produite par les polynucléaires neutrophiles, est présente en grande quantité dans le mucus des malades atteints de mucoviscidose. Cette élastase leucocytaire est, elle aussi, capable de provoquer l'inactivation des IgG par clivage en fragments Fab et Fab'2 [11].

Les alginates possèdent par ailleurs la capacité d'interférer avec les anticorps et d'inhiber la phagocytose en se liant aux macrophages [17]. Ils peuvent inhiber le complément, et leurs propriétés antigéniques déclencheraient un mécanisme d'inflammation accentuant encore la dégradation tissulaire [18].

\section{Les souches mucoïdes de Pseudomonas aeruginosa}

Les souches mucoïdes de Pseudomonas acruginosa présentes dans les poumons des malades atteints de mucoviscidose sont le reflet d'une adaptation particulière, mais parfaite, de la bactérie à son hôte.

Les souches mucoïdes de Pseudomonas aeruginosa se caractérisent par la production de quantités importantes d'exopolysaccharides de type alginate, production pouvant parfois atteindre de 2 à 3 fois le poids de la bactérie. L'observation des souches mucoïdes en microscopie électronique à transmission permet de visualiser, après coloration au rouge de ruthénium (figure 2, p. 891 photo A), les alginates qui entourent le microorganisme et en doublent le volume. Une coloration à l'acide phosphotungstique de ces mêmes bactéries permet de déli- 


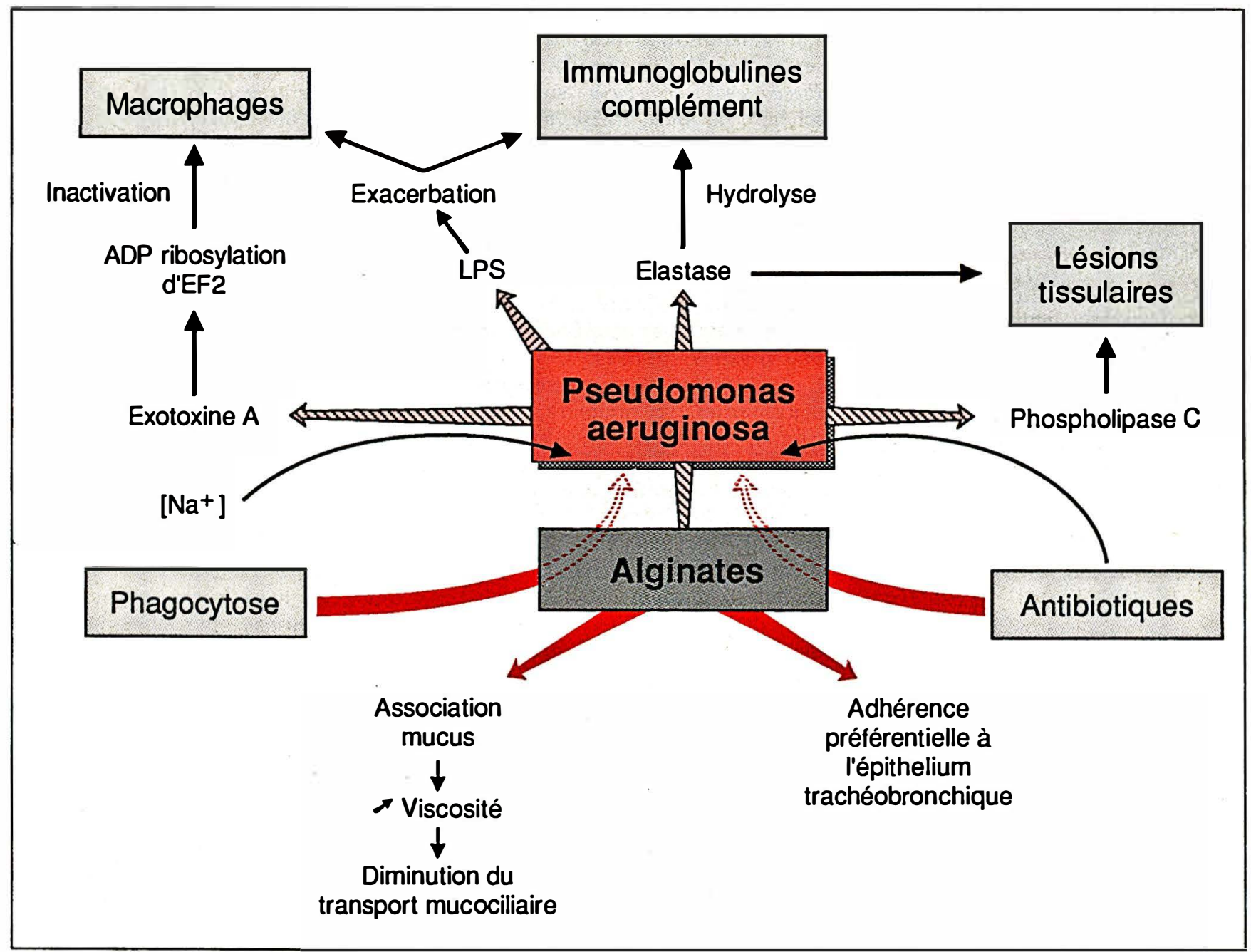

Figure 1. Les relations de Pseudomonas aeruginosa avec son hôte. La bactérie adsorbée sur les cellules trachéales sécrète un certain nombre de facteurs cytotoxiques (partie haute de la figure) et fabrique des alginates qui renforcent son adhérence aux tissus et la protège des systèmes d'élimination (partie basse de la figure). EF2 : facteur d'élongation 2; LPS : lipopolysaccharide de la membrane bactérienne.

miter les structures membranaires, donnant ainsi une idée du volume bactérien moyen, et de visualiser des structures filamenteuses reliant les bactéries entre elles (flèches, figure 2, photo $B)$.

\section{- Les alginates}

Les alginates sont constitués de polymères linéaires d'acide Dmannuronique (M) et d'acide Lguluronique $(G$, son épimère en $C 5)$ en enchaînement B 1-4 (figure 3, p. 891). Ces polymères sont de haut $\mathrm{m} / \mathrm{s} n^{\circ} 9$, vol. 6 , novembre 90 poids moléculaire et peuvent comporter plusieurs centaines de résidus.

D'autres souches de Pseudomonas comme Pseudomonas mendocina et Pseudomonas cepacia sont capables, dans certaines conditions, de produire des alginates, ce qui explique leur apparition en tant qu'éléments pathogènes chez les malades atteints de mucoviscidose et les nouvelles résistances aux antibiotiques observées vis-à-vis de ces dernières souches.

Les alginates produits par Pseudomonas aeruginosa se caractérisent par la présence de groupements acétyls sur certains résidus mannuroniques (M) qui les protègent de l'épimérisation en acides guluroniques (G). La composition en acides uroniques des alginates de Pseudomonas aeruginosa est de type alterné, $\mathrm{MM}$ et $\mathrm{MG}$, avec un rapport de $\mathrm{M} / \mathrm{G}$ toujours supérieur à 1 [14]. L'absence de blocs GG, poly$\mathrm{G}$ ou poly-M est la différence la plus importante entre les alginates de Pseudomonas aeruginosa et ceux d'Azotobacter vinelandii ou des algues brunes Fucus gardneri. 


\section{RÉFÉRENCES}

12. Govan JRW, Harris GS. Pseudomonas aeruginosa and cystic fibrosis : unusual bacterial adaptation and pathogenesis. Microbiol Science $1986 ; 3$ : 301-8.

13. Berry A, De Vault JD, Chakrabarty AM. High osmolarity is a signal for enhanced algD transcription in mucoid and non mucoid Pseudomonas aeruginosa strains. $J$ Bacteriol 1989 ; 171 : 2312-7.

14. Nichols W, Dorrington SM, Slack MPE, Walmsmey HL. Inhibition of tobramycin diffusion by binding to alginate. Antimicrobial Ag Chemother 1988 ; 132 : 518-23.

15. Doig P, Smith NR, Todd T, Irvin RT. Characterization of the binding of $P$. aeruginosa alginate to human epithelial cells. Infect Immun $1987 ; 55: 1517-22$.

16. Jacquot J, Tournier JM, Puchelle E. In vitro evidence that human airways lysozyme is cleaved and inactivated by $P$. aeruginosa elastase and not by human leukocyte elastase. Infect Immun $1985 ; 47$ : 555-60.

17. Simpson JA, Smith SE, Dean RT. Alginate inhibition of the uptake of Pseudomonas aeruginosa by macrophages. $J$ Gen Microbiol $1988 ; 134$ : 29-36.

18. Woods PE, Bryan LE. Studies on the ability of alginate to act as a protective immunogen against infection with the Pseudomonas aeruginosa in animals. I Infect Dis 1985 ; 151 : 581-8.

19. Horan NJ, Jarman T, Dawes F. Studies on some enzymes of alginic acid biosynthesis in Azotobacter vinelandii grown on continuous culture. J Gen Microbiol 1981 ; 129 : 2985-90.

20. Barnejee PC, Vanags RI, Chakrabarty AM, Maitra PK. Alginic acid synthesis in Pseudomonas aeruginosa mutants defective in carbohydrate metabolism. $J$ Bacteriol 1983 ; 155 : 238-45.

21. Darzins A, Frantz B, Vanags R, Chakrabarty $A M$. Nucleotide sequence analysis of the phosphomannose isomerase gene (pmi) of Pseudomonas aeruginosa and comparison with the corresponding Escherichia coli gene manA. Gene $1986 ; 42$ : 293-302.

22. Darzins A, Nixon LL, Vanags $R$, Chakrabarty AM. Cloning of Escherichia coli and Pseudomonas aeruginosa phosphomannose isomerase genes and their expression in alginate negative mutants. $J$ Bacteriol 1985 ;

(16)

- Biosynthèse des alginates

La caractérisation des enzymes présentes dans les souches mucoïdes de Pseudomonas aeruginosa suggère un schéma de biosynthèse similaire à celui observé chez les algues brunes et chez les bactéries productrices d'alginates, Azotobacter sp. et Athrobacter sp. Les études biochimiques menées par Horan et al. [19] et par Barnejee et al. [20] ont permis de reconstituer la chaîne de biosynthèse des alginates par Pseudomonas aeruginosa (figure 4, p. 892).

Cette biosynthèse se décompose en deux parties. La première, non spécifique, utilise une voie du métabolisme secondaire du glucose, la voie d'Etner-Doudoroff. La seconde, spécifique de la biosynthèse des alginates, utilise des enzymes induites spécialement pour la fabrication des exopolysaccharides.

Les étapes spécifiques de la biosynthèse des alginates débutent par la transformation du fructose-6-phosphate en mannose-6-phosphate par la phosphomannose isomérase (PMI). Le gène codant pour la PMI (algA) a été localisé à 34 minutes sur le chromosome bactérien. Il a été cloné sur un plasmide interspécifique et placé sous le contrôle du promoteur tac*. Dans ces conditions, après induction par l'IPTG (Isopropyl thiogalactoside), on trouve une activité PMI dans les bactéries transformées et la synthèse d'un polypeptide de 56000 daltons. Le séquençage du gène $\operatorname{alg} A$ a mis en évidence une phase ouverte de lecture codant pour un polypeptide de 53000 daltons [21].

L'hybridation du gène algA de Pseudomonas aeruginosa avec le gène manA codant pour la phosphomannose isomérase d'Escherichia coli montre qu'il n'existe aucune homologie de séquence entre ces deux gènes; en revanche, il existe des régions fortement homologues dans le gène de Pseudomonas mendocina, de Pseudomonas putida et même d'Azotobacter vinelandii [21].

La réaction catalysée par la PMI est une activité commune à la biosynthèse de l'acide colanique impliqué, chez certaines souches mucoïdes d'Escherichia coli, dans la structure des polysaccharides de la capsule, et à la biosynthèse des alginates chez Pseu- domonas aeruginosa. Le produit du gène manA est capable de complémenter un mutant dans le gène algA de Pseudomonas aeruginosa, et la réciproque a été aussi observée [22].

Le phosphate situé en position 6 sur le mannose est ensuite transféré par la phosphomannose mutase (PMM) en position 1. Peu de renseignements ont été obtenus sur la PMM. Cependant on a remarqué que cette activité enzymatique augmente dans les souches mucoïdes productrices d'alginates. La position du gène sur le chromosome bactérien n'est pas encore précisée. Il semblerait pourtant que le gène codant pour la PMM se trouve dans la région 34 minutes, qui doit contenir tous les gènes codant pour les enzymes cytoplasmiques de la biosynthèse des alginates [23].

L'activation du sucre se fait par la GDP-mannose pyrophosphorylase (GMP) avec l'intervention d'une molécule de GTP. Sà-Corréia et al. [23] ont montré que l'hyperexpression du gène algA codant pour la PMI provoque aussi une augmentation de la quantité de GMP dans les bactéries. Devant l'impossibilité de séparer l'activité PMI et l'activité GMP, ces auteurs suggèrent que le gène algA code pour les deux activités. Il se pourrait donc que le même polypeptide de 56000 daltons possédât les deux activités enzymatiques, situées chacune dans des domaines différents de la protéine et fonctionnant indépendamment l'une de l'autre.

La GDP-mannose déshydrogénase (GMD) oxyde en deux étapes le GDP-mannose en GDP-mannuronate (figure 4). Cette réaction nécessite la réduction de deux molécules de $\mathrm{NAD}^{+}$par molécule de GDPmannose transformée. La GMD est la dernière enzyme cytoplasmique spécifique de la biosynthèse des alginates et sa synthèse est fortement stimulée dans les souches mucoïdes.

Le gène algD codant pour la GMD a été identifié et situé dans la même région chromosomique 34 minutes. Ce gène a été cloné dans un plasmide interspécifique et placé sous le contrôle du promoteur tac*. Son expression dans des "maxicells "** $\mathrm{d}^{\prime} E$. coli conduit à la fabrication d'un polypeptide de 48000 daltons [24]. 


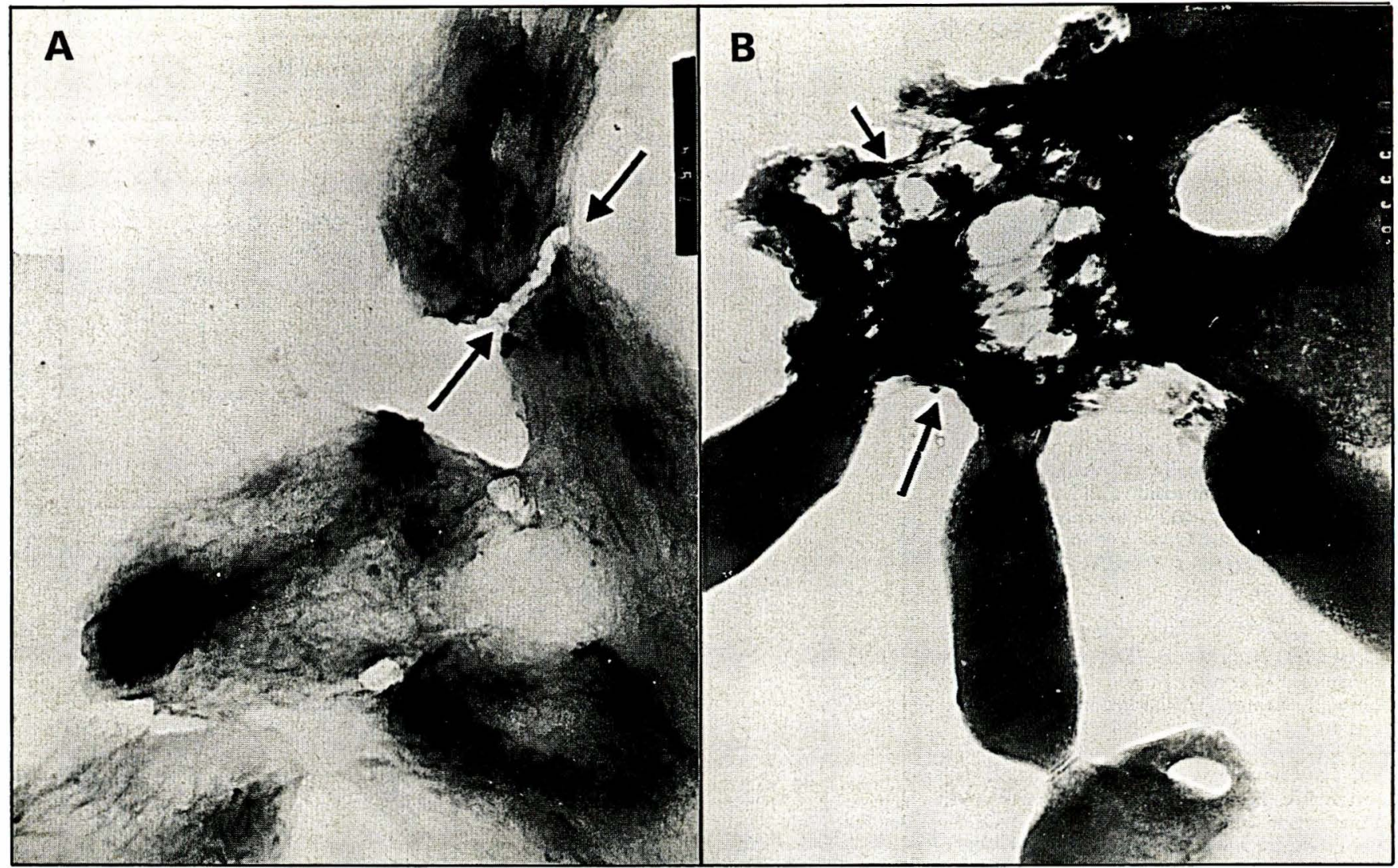

Figure 2. Observation en microscopie électronique à transmission de la souche mucoïde Pseudomonas aeruginosa A22 alg +. (A): coloration des acides uroniques au rouge de ruthénium. Grossissement 50000 . (B) coloration à l'acide phosphotungstique. Grossissement 50 000. Les flèches montrent les structures filamenteuses qui relient les bactéries entre elles et les associent.

La purification de la GMD a été entreprise à partir de souches hyperproductrices contenant le gène algD sous le contrôle du promoteur tac. Dans ces conditions, la GMD se présente sous forme d'un hexamère de 290000 daltons constitué par l'association de 6 sous-unités identiques de

- Promoteur tac : promoteur obtenu par la fusion de la région d'ADN - 35 du promoteur de l'opéron tryptophane avec la région - 10 et l'opérateur de l'opéron lactose. Ce promoteur hybride est controle par le répresseur de l'opéron lactose.

** "Maxicells ": cellules obtenues après une forle irradiation à $254 \mathrm{~nm}$ de bactéries Escherichia coli deficionles dans les systimes de recombinaison (RecA) et de photoréparation (UvrA et Phr1). L'ADN chromosomique n'est plus transcrit tandis qu'un $A D N$ plasmidique est encore exprimé.

$m / s{ }^{\circ} 9$, ool. 6 , noocmbre 90

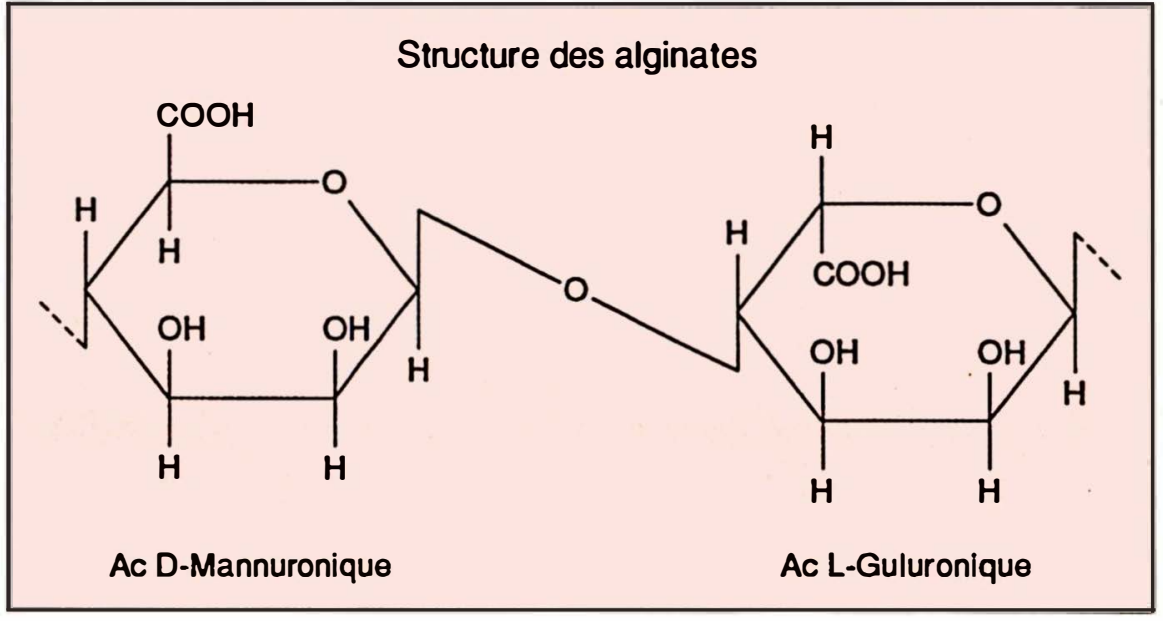

Figure 3. Structure des alginates. Les alginates sont constitués d'acides $D$ mannuroniques $(M)$ et $d^{\prime}$ acides $L$-guluroniques $(G)$ en enchaînement $B 1 \rightarrow 4$. 


\section{RÉFÉRENCES}

23. Sà-Corréia I, Darzins A, Wang SK, Berry A, Chakrabarty AM. Alginate biosynthesis in mucoid and non mucoid Pseudomonas aeruginosa : overproduction of PMI, PMM and GMP by overexpression of the pmi gene. $J$ Bacteriol 1987; 169 : 3224-31

24. Deretic V, Gill JF, Chakrabarty AM Gene algD coding for GDP-mannose dehydrogenase is transcriptionnally activated in mucoid strains. J Bacteriol 1987; 169 : 351-8.

25. Roychoudhury S, May TB, Gill JF, Singh SK, Feingold DS, Chakrabarty AM. Purification and characterization of GDPmannose dehydrogenase. J Biol Chem 1989 ; 264 : 9380-5.

26. Chitnis CE, Ohman DE. Cloning of $P$. anuginosa algG, which controls alginate structure. J Bacteriol $1990 ; 172$ : 2894-900.

27. Nguyen L, Schiller N. Identification of a slime polysaccharide depolymerase in mucoid strains of Pseudomonas acruginosa. Current Microbiol 1989 ; 18 : 323-9.

28. Flynn JL, Ohman DE. Use of gene replacement cosmid vector for cloning alginate conversion genes from mucoid and non mucloid Pseudomonas aeruginosa strains : algS controls expression of algT. J Bacteriol 1988 ; $170: 3228-36$.

29. Deretic V, Konyecsni WM, Mohr CD, Martin DW, Hiber NS. Common denominators of promoter control in Pseudomonas and other bacteria. Bio/Technology $1989 ; 7$ : 1249-54.

30. Konyecsni WM, Deretic V. DNA sequence and expression analysis of algP and al $g Q$ components of the multigene system transcriptionally regulating mucoidy in $P$. aeruginosa : algP contains direct repeats. $J$ Bacteriol 1990 ; 172 : 2511-20.

31. Kimbara K, Chakrabarty AM. Control of alginate synthesis in Pseudomonas aeruginosa : regulation of the algR1 gene. Biochem Biophys Res Comm 1989 ; 164 : 601-8.

32. Kato J, Misra TK, Chakrabarty AM. AlgR 3, a protein resembling eukaryotic histone $\mathrm{H} 1$, regulates alginate synthesis in $P$. aenuginosa. Proc Natl Acad Sci USA 1990 ; 87 : 2887-91.

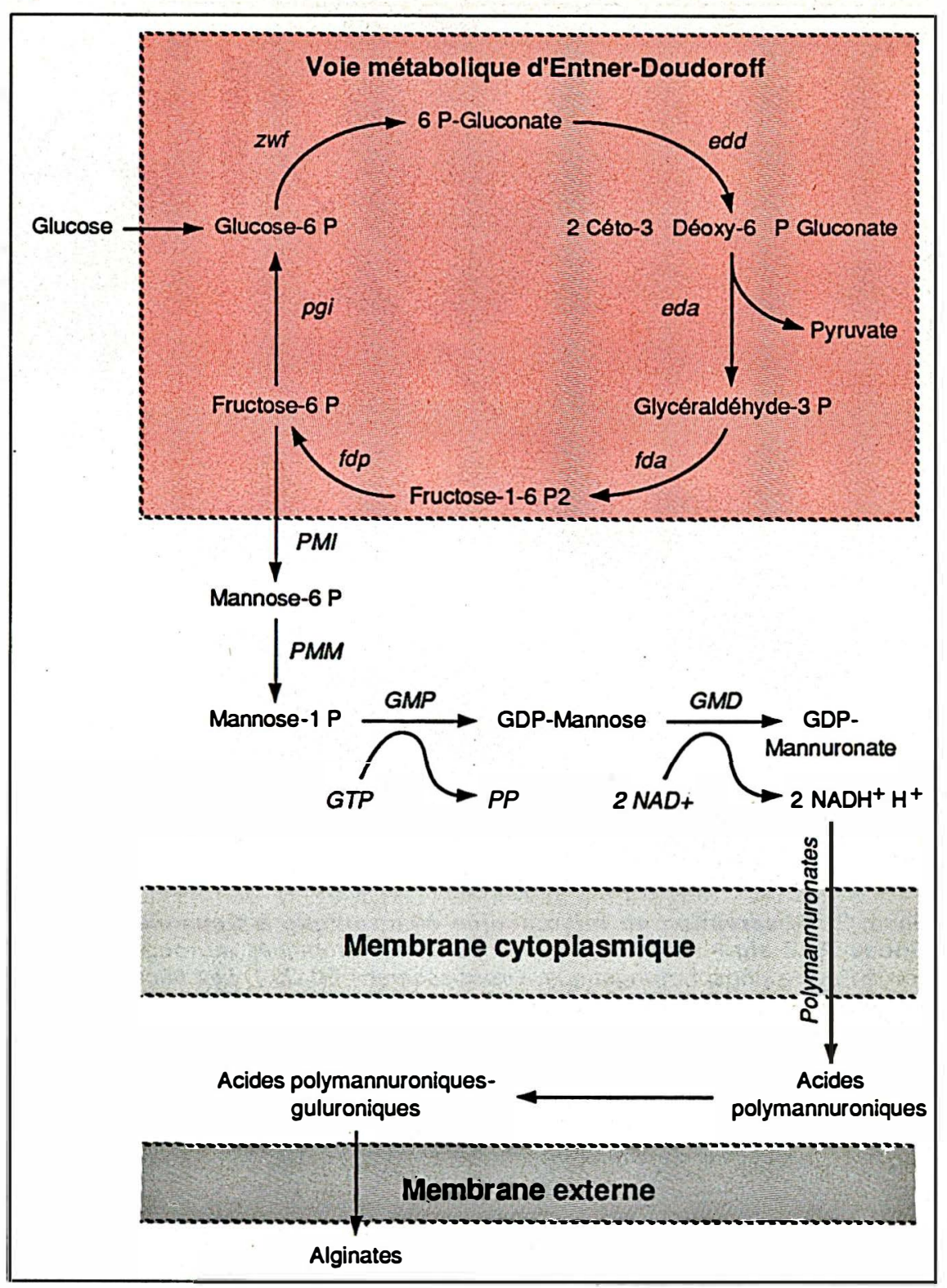

Figure 4. Schéma de biosynthèse des alginates chez Pseudomonas aeruginosa. zwf: glucose 6-phosphate déshydrogénase, edd: phospho-gluconate déhydratase, eda : phospho-2céto-3désoxy gluconate aldolase, fda : fructose diphosphate aldolase, fdp : fructose diphosphatase, pgi : phosphoglucose isomérase, PMI : phospho-mannose isomérase, PMM : phospho-manno-mutase, GMP: GDP-mannose pyrophosphorylase, GMD : GDP-mannose déshydrogénase. 
48000 daltons [25]. Dans les souches naturelles mucoïdes, la protéine se trouve sous forme d'un monomère auquel est associé au moins un nucléosido-sucre.

Les étapes extracytoplasmiques de la biosynthèse des alginates chez Pseudomonas aeruginosa (polymérisation, acétylation, épimérisation) sont très mal connues et aucune des protéines qui participent à ces étapes n'a été isolée. Cependant, très récemment, le gène alg $G$ qui code pour la $\mathrm{C} 5$ épimérase, enzyme qui transforme les acides D-mannuroniques non acétylés en acides L-guluroniques, a été cloné et localisé dans la région 34 minutes entre $\operatorname{alg} D$ et $\operatorname{alg} A$ [26]. La caractérisation du produit de ce gène apporterait des renseignements précieux sur les dernières étapes de la biosynthèse des alginates.

Un dernier type d'activité associée aux fractions membranaires et périplasmiques doit être signalé. Les souches mucoïdes et non mucoïdes de Pseudomonas aeruginosa possèdent une activité exopolysaccharide dépolymérase surtout active sur les acides poly-mannuroniques non acétylés [27]. L'intégration de ce type d'activité dans le schéma de biosynthèse ne semble pas aisée, sauf si l'on veut bien considérer que la mesure de ces activités lytiques pourrait représenter la réaction inverse de la polymérisation.

\section{- Régulation de la biosynthèse des} alginates

L'apparition de la mucoïdie chez les souches de Pseudomonas aeruginosa est un phénomène complexe, sous la dépendance de facteurs externes à la bactérie. Plusieurs études génétiques ont montré que toutes les souches de Pseudomonas aeruginosa possédaient l'information nécessaire à la synthèse des enzymes intervenant dans la biosynthèse des alginates.

Trois régions du chromosome semblent impliquées dans le passage des souches vers la forme mucoïde. Deux régions de régulation, une à 9 minu-

' tes sur la carte chromosomique, l'autre à 68 minutes, et une région contenant les gènes de structure pour les enzymes impliquées dans la chaîne de biosynthèse, et située à 34 minutes.

Bien que l'on puisse dresser un $m / s n^{\circ} 9$, ool. 6 , noombre 90 schéma de régulation (figure 5), on ne sait pas actuellement quel est l'élément premier qui déclenche le phénomène, ni sur quoi il agit. Cependant, il semble bien qu'un rôle important soit tenu par la région 68 minutes du chromosome, où sont situés les gènes algS et alg $T$ qui contrôlent le passage des souches non mucoïdes vers la forme mucoïde. algS contrôle l'expression du gène algT, dont le produit diffusible agit sur une cible non encore identifiée [28].

La région de régulation 9 minutes a été beaucoup plus étudiée, et on y a décelé la présence de cinq gènes de régulation, algP, algQ algR1, algR2 et algR3 [29-32]. Les produits des gènes algQ algR1 et algR2 agissent sur le promoteur du gène algD qui code pour la GDP-mannose déshydrogé- nase et activent la transcription du gène, ce qui explique les grandes quantités d'enzyme trouvées dans les souches mucoïdes.

L'activation de la transcription par

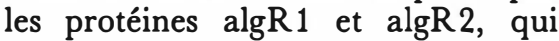
agissent conjointement, est en relation directe avec la concentration en sel du milieu extérieur. En effet, le gène algR1 ressemble au gène ompR de régulation de la biosynthèse des protéines de membrane externe chez Escherichia coli, et des expériences ont montré que la protéine ompR était capable de stimuler la transcription du gène $\operatorname{alg} D$. Ces gènes de régulation font partie de la classe des gènes activés en réponse à une modification de l'osmolarité du milieu extérieur, et l'augmentation de la concentration en $\mathrm{NaCl}$ se traduit par une augmen-

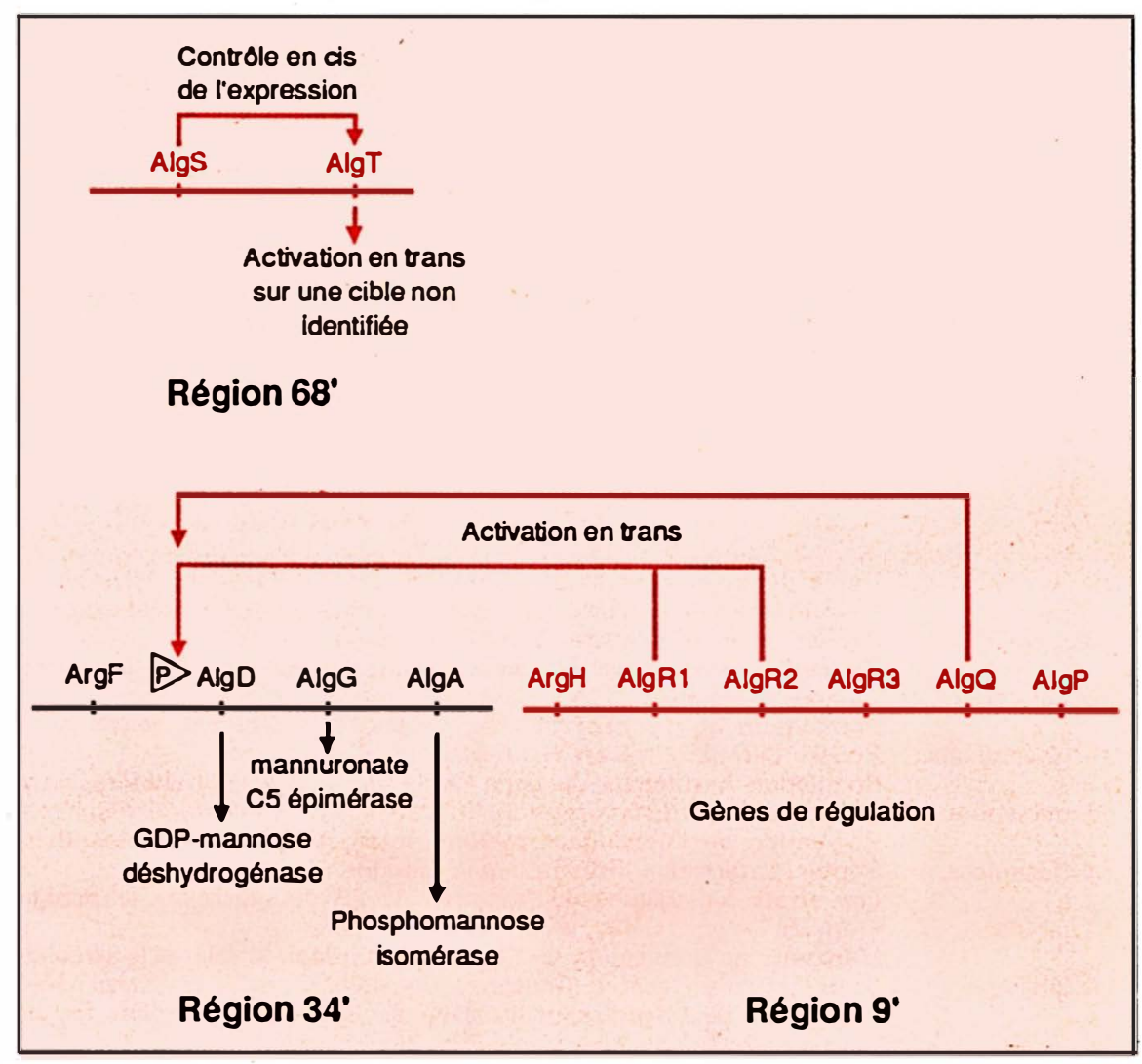

Figure 5. Régulation de la biosynthèse des alginates chez les souches mucoïdes de Pseudomonas aeruginosa. 
tation du niveau de synthèse de la GDP-mannose déshydrogénase [13]. Tous les résultats obtenus jusqu'à maintenant concernent l'activation du gène algD. En revanche, le contrôle de l'expression des autres gènes de structure de la région 34 minutes n'est pas encore élucidé.

Le quatrième gène identifié dans la région 9 minutes, algP, présente du côté 3' une répétition directe de séquences en tandem, six unités de 75 paires de bases. Le gène algP paraît agir en synergie avec algQ les deux gènes étant exprimés de manière constitutive chez toutes les souches de Pseudomonas aeruginosa, qu'elles soient mucoïdes ou non mucoïdes [30].

Le produit du gène algR3 possède toutes les propriétés des protéines de type histone, avec une moitié Cterminale riche en lysines au sein de courtes séquences ala-ala-lys-pro répétées une trentaine de fois [32]. Ce même motif peut d'ailleurs être retrouvé dans la protéine hypothétique (car non encore caractérisée) correspondant à la phase ouverte de lec- ture du gène algP, où on le trouve répété 36 fois du côté $\mathrm{C}$ terminal [30].

L'étude de ce schéma de régulation permet de mieux comprendre l'apparition de la mucoïdie chez les souches de Pseudomonas aeruginosa et le rôle important joué par la concentration anormalement élevée en $\mathrm{Na}+$ et $\mathrm{Cl}$ - dans le mucus des poumons des malades, comme inducteur de l'activation des gènes de régulation, puis des gènes de structure des protéines qui participent à la biosynthèse des alginates. La présence de protéines de type histone parmi les produits des gènes de régulation pose le problème de la structure de l'ADN au niveau des promoteurs. Une régulation par variation de la topologie de la molécule d'ADN au niveau des promoteurs, en particulier alg $\mathrm{D}$ et algR1, pourrait ainsi intervenir

\section{TIRÉS A PART}

C. Hulen.

\section{Summary}

Colonization and persistence of mucoid strains of Pseudomonas aeruginosa in cystic fibrosis patients' lungs

Chronic lung infection by Pseudomonas aeruginosa is the most important cause of morbidity and mortality in cystic fibrosis (CF) patients.

High osmolarity of the mucus activates transcription of $P$. aeraginosa genes that control alginate biosynthesis. Bacteria are then protected from both antibiotics and normal lung clearance systems, i.e. phagocytosis and ciliary activity. In addition, bacterial toxine release results in alteration of the tracheal epithelial cells and in hydrolysis of some components of the humoral defense systems. Alginate also increases viscosity of the bronchial mucus, resulting therefore in respiratory tract obstruction. 